\title{
A Simplification of Maxwell's Equations in Conformity with the Flux-cutting Principle
}

\author{
By Dr. C. V. Drysdale, C.B., O.B.E.
}

$\mathrm{D}$ URING the last thirty years, the question as to whether electromagnetic induction is caused by the change or by the cutting of magnetic flux has been debated at intervals. Faraday's rotating disk experiment and the later experiments of Blondel ${ }^{1}$ and of Messrs. Cramp and Norgrove ${ }^{2}$, as well as Prof. H. A. Wilson's experiments with moving dielectrics ${ }^{3}$, all appear definitely to favour the fluxcutting hypothesis; and Rowland's experiments with revolving electrostatically charged disks ${ }^{4}$ indicated that a magnetic force is similarly induced by the cutting of an electric field. There seems little doubt that Faraday himself believed that electromagnetic induction was produced by the cutting of his magnetic "lines".

The four fundamental equations of Maxwell for a non-conducting dielectric medium :
(i) $4 \pi D=\varepsilon E$,
(iii) $-\operatorname{curl} E=\dot{B}$,
(ii) $B=\mu H$,
(iv) curl $H=4 \pi \dot{D}$,

(where $E$ and $H$ are the electric and magnetic forces, $D$ and $B$ the corresponding displacement and induction; and $\varepsilon$ and $\mu$ the dielectric constant and permeability of the medium, respectively) were avowedly intended to put Faraday's concepts into mathematical form; but the two latter equations are obviously based on the change of flux principle, as they give the line integrals of the electric or magnetic forces around unit area due to the rate of change of magnetic or electric flux through that area, and not the actual forces themselves. These two equations are not only difficult to comprehend and to visualize physically, but also they are difficult of application owing to the complex differential equations to which they give rise; and, if the flux-cutting principle is the true one, they are not completely fundamental.

It is easy to show that a simple transformation of the Maxwellian equations divests them of all complexity and harmonizes them with the flux-cutting principle. For if we write $j \frac{\partial}{\partial x}$ for curl, equation (iii) becomes

$$
-j \frac{\partial E}{\partial x}=\frac{\partial B}{\partial t}=\frac{\partial B}{\partial x} \frac{d x}{d t}=V \frac{\partial B}{\partial x},
$$

from which $E=j V B$, if $E=0$ when $V=0$. This is equivalent to the dynamo designer's formula E.M.F. = $l V B$ for the electromotive force in a conductor of length $l$ moving transversely with velocity $V$ across a magnetic field $B$, since the electric force $E$ is the electromotive force per unit length. The quadrantal versor $j=\sqrt{-1}$ simply implies that the electric force is perpendicular to the plane containing the motion and magnetic flux, and does not affect its magnitude.

Similar treatment of equation (iv) gives us $H=-j 4 \pi V D$, which is obviously proportional to the rate of cutting the electrostatic flux and is in accordance with the results of Rowland's experiments ; and if we harmonize the electrostatic and magnetic units by writing $J$ for $4 \pi D$, which implies $4 \pi$ electrostatic "lines" of induction per unit charge, in conformity with $4 \pi$ magnetic lines per unit pole, Maxwell's equations become :
(i) $J=\varepsilon E$,
(iii) $E=j V B$,
(ii) $B=\mu H$,
(iv) $H=-j V J$,

for an isotropic dielectric medium moving with velocity $V$, or :

$$
\begin{array}{ll}
\text { (ia) } J=\varepsilon E, & \text { (iii } a) E=-j V B, \\
\text { (iia) } B=\mu H, & \text { (iva) } H=j V J,
\end{array}
$$

for a stationary isotropic dielectric and moving fields. The change of sign in the last two equations is equivalent to the change from the 'right hand' to the 'left hand' rule in changing from moving armature to moving field machines.

These equations are all of the same simple linear form, and are easily grasped, memorized and handled, since the last two may be summed up as :

Induced $\left\{\begin{array}{c}\text { electric } \\ \text { magnetic }\end{array}\right\}$ force is equal to the rate of cutting $\left\{\begin{array}{c}\text { magnetic } \\ \text { electric }\end{array}\right\}$ lines.

\section{Applications to Electromagnetic Wave PROPAGATION}

A few applications to the theory of electromagnetic wave propagation will serve to show their convenience. Combining (iia) and (iiia), we have $E=-j \mu V H$ : and combining (i $a$ ) and (iva), $H=j \varepsilon V E$. If we suppose the north pole of a magnet moving trans. versely through an isotropic non-conducting medium with velocity $V$, as shown in the accompanying

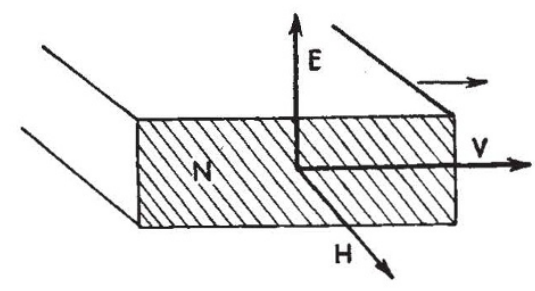

diagram, its magnetic force $H$ will be directed horizontally forward, and according to the first of these combined equations, it will induce an electric force in the medium, $E=-j \mu V H$, directed vertically upwards. By the second combined equation, this electric force will in turn induce a magnetic force $H^{1}=j \varepsilon V E=\varepsilon \mu V^{2} H$, since $j^{2}=-1$, so that $H^{1}$ will be in the same direction as $H$, and will therefore tend to strengthen the magnet, just as a capacitance load on an alternator causes a rise of E.M.F. by strengthening its magnetic field. The transverse motion of the magnet therefore produces a forced electromagnetic disturbance or wave in the medium, and as the velocity of the magnet is increased the 
magnetic and electric fields will increase, unless $H$ is diminished to $H-H^{1}=\left(1-\varepsilon \mu V^{2}\right) H$, as an engineer reduces the excitation of an alternator to prevent the rise of voltage with a leading current. When $\varepsilon \mu V^{2}=1$, or $V=1 / \sqrt{\varepsilon \mu}$, therefore, $H$ should be reduced to zero, or the magnet may be withdrawn, and the forced electromagnetic wave will persist as a free one in which $E$ and $H$ are maintained by their reciprocal inductive interactions, and it will travel forward unchanged with the velocity $V=1 / \sqrt{\varepsilon \mu}$, which is Maxwell's expression for the velocity of propagation of electromagnetic waves, including light. Since $E=-j \mu V H$, and $V=1 / \sqrt{\varepsilon \mu}, E=-j \sqrt{\mu / \varepsilon} . H$ : so that as $\varepsilon$ and $\mu$ are constants for all dielectric media, $E$ and $H$ must have a constant ratio and hence have the same wave-form and phase.

The formula $E=-j V B=-j \mu V H$, which represents the rate of cutting magnetic lines, is already familiar to scientific and technical students, but the corresponding formula $H=j V J=j \varepsilon V E$, which represents the rate of cutting electric lines, is not; so that it may be well to show that it is in conformity with known elementary principles. A vertical electric force $E$ in a dielectric medium produces an upward electrical displacement $D$ such that $4 \pi D=\varepsilon E$, so that if we consider the magnetic field $H$ as stationary while the medium is flowing past it with velocity $V$ in the opposite sense, the effect of this displacement will be that of two horizontal surfaces, an upper one charged with surface density $+D$, and a lower one charged with density $-D$, both moving with the medium from right to left. The upper surface will be equivalent to a current sheet of intensity $V D$ per unit breadth flowing from right to left, and the lower to a similar current sheet flowing in the opposite sense since it is negatively charged; so that the total effect will be that of a current sheet or solenoid of intensity $V D$ per unit length circulating round the region of electric displacement in a counter-clockwise direction, which must produce a magnetic force $H$ in that region equal to $4 \pi V D=\varepsilon V E$ and directed horizontally forward, and hence $H=-j \varepsilon V E=-j V J$, the negative sign being due to our dealing in this case with a moving medium instead of moving fields. Since for a moving medium $E=j V B=j \mu V H=$ $\varepsilon \mu V^{2} E, E$ and $H$ will be mutually maintained when the velocity of the medium $V=1 / \sqrt{\varepsilon \mu}$, so that if an electromagnetic disturbance is once started, it will persist unchanged in magnitude and position and will therefore be travelling with the velocity $1 / \sqrt{\varepsilon \mu}$ relatively to the moving medium. This is analogous to Prof. P. G. Tait's well-known simple demonstration of the propagation of transverse waves along stretched cords or belts.

Crystalline Media. The above equations apply for all isotropic non-conducting dielectrics, but in crystalline media $\varepsilon$ varies with the orientation of $E$, so that the direction of $D$ or $J$ is not in general identical with that of $E$ or perpendicular to the plane of $B$ and $V$; and as it is only the component of $J$ perpendicular to $V$ which is concerned in the cutting and the induction of $H$, we must write $H=j V J \sin \theta$, or $[V J]$, where $\theta$ is the angle between $J$ and $V$, and the square brackets imply the vector product of $V$ and $J$. Similarly we may write $E=-[V B]$, but since the permeability of dielectric media does not differ materially in any direction from that of space, $B$ is simply $\mu H$ and is always in the direction of $H$ and perpendicular to $V$, so that we may write $E=-j V B$ or $-[V B]$ as we prefer.
Hence the fundamental equations for all dielectric media are :

$J_{1}=\varepsilon_{1} E_{1}$ etc., $B=\mu H, E=-[V B]$, and $H=[V J]$, which lead to the following six simple algebraical equations :

$$
\begin{aligned}
& E_{1}=V_{3} B_{2}-V_{2} B_{3}=\mu\left(V_{3} H_{2}-V_{2} H_{3}\right) \\
& E_{2}=V_{1} B_{3}-V_{3} B_{1}=\mu\left(V_{1} H_{3}-V_{3} H_{1}\right) \\
& E_{3}=V_{2} B_{1}-V_{1} B_{2}=\mu\left(V_{2} H_{1}-V_{1} H_{2}\right) \\
& H_{1}=V_{2} J_{3}-V_{3} J_{2}=\varepsilon_{3} V_{2} E_{3}-\varepsilon_{2} V_{3} E_{2} \\
& H_{2}=V_{3} J_{1}-V_{1} J_{3}=\varepsilon_{1} V_{3} E_{1}-\varepsilon_{3} V_{1} E_{3} \\
& H_{3}=V_{1} J_{2}-V_{2} J_{1}=\varepsilon_{2} V_{1} E_{2}-\varepsilon_{1} V_{2} E_{1}
\end{aligned}
$$

where the suffixes imply the components in the three axes of the crystal.

Combining these we have :

$$
\begin{aligned}
& \left\{1-\varepsilon_{1} \mu\left(V_{2}^{2}+V_{3}^{2}\right)\right\} E_{1}+\varepsilon_{2} \mu V_{1} V_{2} E_{2}+\varepsilon_{3} \mu V_{1} V_{3} E_{3}=0 \\
& \left\{1-\varepsilon_{2} \mu\left(V_{1}^{2}+V_{3}^{2}\right)\right\} E_{2}+\varepsilon_{3} \mu V_{2} V_{3} E_{3}+\varepsilon_{1} \mu V_{1} V_{2} E_{1}=0 \\
& \left\{1-\varepsilon_{3} \mu\left(V_{1}^{2}+V_{2}^{2}\right)\right\} E_{3}+\varepsilon_{1} \mu V_{1} V_{3} E_{1}+\varepsilon_{2} \mu V_{2} V_{3} E_{2}=0
\end{aligned}
$$

Hence if the propagation is along axis $1, E=0$, $\left(1-\varepsilon_{2} \mu V_{1}^{2}\right) E_{2}=0$, and $\left(1-\varepsilon_{g} \mu V_{1}{ }^{2}\right) E_{3}=0$, which show that the components $E_{2}$ and $E_{3}$ travel with the velocities $1 / \sqrt{\varepsilon_{2} \mu}$ and $1 / \sqrt{\varepsilon_{3} \mu}$ respectively, so that they must be regarded as separate waves polarized in perpendicular planes. Similarly for propagation along axes 2 and 3 , and hence the wave surface must have two sheets intersecting the three axes at radii $a$ and $b, a$ and $c$, and $b$ and $c$ respectively, where $a=1 / \sqrt{\varepsilon_{1} \mu}, b=1 / \sqrt{\varepsilon_{2} \mu}$, and $c=1 / \sqrt{\varepsilon_{3} \mu}$.

It should be noted that since the directions of $J$ and $E$ are not in general the same, the wave cannot be self-maintained unless $E, J$ and $V$ are coplanar, as if $J$ is deviated out of the plane $E V, H=[V J]$ must be perpendicular to the plane $J V$ and the induced electric force $E_{1}=-\mu[V H]$ will be in the plane $J V$ instead of the plane $E V$ and cannot be coincident with $E$ for any value of $V$. But for any direction of $E$ there will be a plane containing $E$ and $J$, so that if $V$ is in this plane and perpendicular to $E$, the induced electric force will be identical with $E$ for a certain value of $V$, and hence a plane polarized beam can be transmitted in that plane. For $E, J$ and $V$ to be coplanar the scalar product $(E J V)=\left(\varepsilon_{3}-\varepsilon_{2}\right) E_{2} E_{3} V_{1}+$ $\left(\varepsilon_{1}-\varepsilon_{3}\right) E_{1} E_{3} V_{2}+\left(\varepsilon_{2}-\varepsilon_{1}\right) E_{1} E_{2} V_{3}$ must be zero, and it is easy to show that this condition can always be satisfied for two perpendicular planes. Hence for any direction of propagation the electric force will be resolved into two perpendicular components both of which will be self-maintaining or transmitted, but in general with different velocities, so that the medium will be doubly refracting and the two components will be plane polarized in perpendicular planes.

Semi-Conducting Dielectrics. In such media an electric force $E$ produces a steady current of density $\dot{D}=\dot{J} / 4 \pi=g E$, where $g$ is the conductivity, in addition to $J=\varepsilon E$, and hence $J=\varepsilon E+4 \pi g E$, where $E=$ $\int E d t$. Hence for all isopic semi-conducting media :

$$
J=\varepsilon E+4 \pi g E, B=\mu H, E=-j V B \text {, and } H=j V J,
$$

and the induced electric force $E^{1}=\mu V^{2}(\varepsilon E+4 \pi g E)$, which must balance the impressed electric force $E$ for a self-maintained wave. But the power dissipated in the conductance causes an attenuation or reduction of $E$ as the wave travels forward, and since the displacement of the dissociated ions depends on the previous variations of the electric force, we shall only 
obtain the steady periodic condition by differentiating twice with respect to time, and writing $\frac{d^{2} E^{1}}{d t^{2}}=$ $\mu V^{2}(\varepsilon E+4 \pi g \dot{E})$. The variations on the right-hand side depend simply upon the changes of $E$ with time, irrespective of the motion of the wave in space, but the induced electric force $E^{1}$ is caused by the cutting due to the motion and we must therefore write

$$
\frac{d E^{1}}{d t}=\frac{\partial E^{1}}{\partial x} \frac{d x}{d t}=V \frac{\partial E^{1}}{\partial x}, \text { and } \frac{d^{2} E^{1}}{d t^{2}}=V^{2} \frac{\partial^{2} E^{1}}{\partial t^{2}},
$$

which gives us $\frac{\partial^{2} E}{\partial x^{2}}=\varepsilon \mu \frac{\partial^{2} E}{\partial t^{2}}+4 \pi g \mu \frac{\partial E}{\partial t}$ for a selfmaintained wave.

This relation arises directly from the Maxwellian equations, but the above treatment is not only more simple but also gives a clear idea of the physical processes involved, and it directs attention to the question of the actual displacement of the dissociated ions which may give rise to transients.

Lorentz Theory of Moving Media. According to the ionic constitution of matter, the absolute dielectric constant of a medium is $\varepsilon_{0}+\varepsilon_{i}$, where $\varepsilon_{0}$ is the absolute dielectric constant of space, and $\varepsilon_{i}$ that due to the displacement of the ions, which is equal to $4 \pi \Sigma \frac{N e^{2}}{K-m \omega^{2}+j b \omega}$, where $N$ is the number of ions of charge $e$, mass $m$, coupling constant $k$, and damping coefficient $b$ per unit volume, for a sinusoidal disturbance for which $\omega$ is $2 \pi$ times the frequency; and this in conjunction with the above equations leads to Drude's theory of dispersion. But if the medium is moving and carrying the ions along with it with a velocity $v$ in the direction of propagation, an electromagnetic disturbance travelling with velocity $V$ only travels with velocity $V-v$ relatively to the ions. The conditions are therefore similar to those in an induction motor in which the revolving field cuts the stationary stator bars with a velocity $V$ and the revolving rotor bars with velocity $V-v$, the former being represented by the stationary ether between the ions, and the latter by the moving ions. If a magnetic field $H$ is moving with velocity $V$ through the medium, it will induce an electric force $E_{0}=-j \mu V H$ acting on the stationary ether, and $E_{i}=-j \mu(V-v) H$ acting on the ions; and hence $J_{0}=-j \varepsilon_{0} \mu V H$, and $J_{i}=-j \varepsilon i \mu(V-v) H$. These electric inductions will in turn induce magnetic forces $H_{0}=j V J_{0}=\varepsilon_{0} \mu V^{2} H$ in the ether and $H_{i}=$ $\varepsilon_{i} \mu(V-v)^{2} H$ due to the ions, so that the total induced magnetic force will be $H^{1}=H_{0}+H_{i}=$ $\mu\left\{\varepsilon_{0} V^{2}+\varepsilon_{i}(V-v)^{2}\right\} H$, and the wave will be self. maintaining if $H^{1}=H$, or $\varepsilon_{0} \mu\left\{V^{2}+\frac{\varepsilon_{i}}{\varepsilon_{0}}(V-v)^{2}\right\}=1$. But $\frac{\varepsilon_{0}+\varepsilon_{i}}{\varepsilon_{0}}=1+\frac{\varepsilon_{i}}{\varepsilon_{0}}$ is the ordinary relative or measured dielectric constant of the medium $\varepsilon=n^{2}$, where $n$ is the refractive index of the medium for the frequency of the disturbance, and hence $\varepsilon_{i} / \varepsilon_{0}=$ $n^{2}-1$ and $V^{2}+\left(n^{2}-1\right)(V-v)^{2}=1 /\left(\varepsilon_{0} \mu\right)=c^{2}$, where $c$ is the velocity of propagation in space.

To the first order of $v / V=n v / c$, this gives us :

$$
V=\frac{c}{n}+\frac{n^{2}-1}{n^{2}} v
$$

if the source is moving with the medium, or

$$
V=\frac{c}{n}+\left(\frac{n^{2}-1}{n^{2}}-\frac{\lambda}{n} \frac{\partial n}{\partial \lambda}\right) v
$$

for a stationary source and moving medium.

These are Lorentz's relations for the propagation of electromagnetic waves through moving dispersive media, which have been confirmed by the movingwater experiments of Zeeman and give a theoretical basis for the Fresnel drag coefficient $\left(n^{2}-1\right) / n^{2}$.

The above applications will suffice to show that these simple equations lead to results which are in agreement with those derived from the Maxwellian equations, and their simplicity and fundamental correctness of principle may lead to useful extensions, especially as they focus attention on the physical interactions involved. For closed circuits of rigid conformation where we are concerned only with the total induced electromotive force, the ordinary rateof-change or 'transformer' formula $E=\partial N / \partial t$ is correct and more convenient, and even for deform. able circuits I have shown ${ }^{5}$ from energy considerations that the most convenient general formulæ for calculating the force or torque in such systems are $f=\frac{1}{2} i^{2} \frac{\partial L}{\partial x}$ or $T=\frac{1}{2} i^{2} \frac{\partial L}{\partial \theta}$, where $f$ is the force, $T$ the torque, and $L$ the inductance of the circuit; but it seems to be proved beyond doubt that electric and magnetic forces can only be induced by the cutting of magnetic or electrostatic fields, so that even in closed circuits it appears that any change of flux must involve the actual cutting of magnetic or electrostatic "lines", although it may be difficult to visualize the process in many cases. The simplified Maxwellian equations, based on the flux-cutting or 'dynamo' principle, appear to be the fundamentally correct ones; and, as they are much more simple to handle and comprehend in dealing with radiation problems, it would seem that they should be adopted.

\section{SUMMARY}

Faraday's rotating disk experiment and the later experiments of Blondel, Cramp and Norgrove, and H. A. Wilson all indicate that magnetoelectric induction is caused by the cutting of a magnetic field rather than by its rate of change; and Rowland's experiments with rotating electrostatically charged disks similarly indicate that a magnetic force is induced by the cutting of an electrostatic field. The fundamental Maxwellian equations are based on the change of flux principle, but it is here shown that a simple transformation of these equations brings them into harmony with the flux-cutting principle and reduces them to four simple algebraic or vector equations which enable radiation problems to be treated with great simplicity and easy grasp of the physical processes involved. Application of these equations to the propagation of electromagnetic waves in isotropic and crystalline dielectries and in semi-conducting and moving media show that they lead to the same results as the Maxwellian equations.

Comptes rendus, 159, 674-679 (1914). Electrician, 75, 341 (1915)

${ }^{2} J$. Inst. Elect. Eng., 78, No. 472, 481 (April 1936).

s Phil. Trans. Roy. Soc., A, 204, 121 (1905).

4 Phil. Mag., (v), 27, No. 169 (June 1889).

5. J. Sci. Instruments, 9, 209-216 (1932). J. Inst. Elect. Eng., 72 365-384 (1933). 